\title{
Amlexanox and Forskolin Prevents Isoproterenol-Induced Cardiomyopathy by Subduing Cardiomyocyte Hypertrophy and Maladaptive Inflammatory Responses
}

\section{OPEN ACCESS}

Edited by:

Susanne Sattler,

Imperial College London, United Kingdom

Reviewed by:

Gobinath Shanmugam, University of Alabama at Birmingham, United States Mohamed Fizur Nagoor Meeran, United Arab Emirates University,

United Arab Emirates

*Correspondence:

Hong Sun

sunh@xzhmu.edu.cn

Specialty section:

This article was submitted to Molecular and Cellular Pathology,

a section of the journal Frontiers in Cell and Developmental

Biology

Received: 02 June 2021

Accepted: 30 August 2021

Published: 24 September 2021

Citation:

Adzika GK, Hou H, Adekunle AO, Rizvi R, Adzraku SY, Li K, Deng Q-M, Mprah R, Ndzie Noah ML,

Adu-Amankwaah J, Machuki JO, Shang W, Ma T, Koda S, Ma X and

Sun $H$ (2021) Amlexanox and Forskolin Prevents Isoproterenol-Induced

Cardiomyopathy by Subduing Cardiomyocyte Hypertrophy and Maladaptive Inflammatory

Responses

Front. Cell Dev. Biol. 9:719351. doi: 10.3389/fcell.2021.719351
Gabriel Komla Adzika1 , Hongjian Hou ${ }^{1,2}$, Adebayo Oluwafemi Adekunle1, Ruqayya Rizvi , Seyram Yao Adzraku ${ }^{4}$, Kexue Li1 , Qi-Ming Deng ${ }^{5}$, Richard Mprah', Marie Louise Ndzie Noah'1, Joseph Adu-Amankwaah'1, Jeremiah Ong'achwa Machuki', Wenkang Shang ${ }^{6}$, Tongtong $\mathrm{Ma}^{1}$, Stephane Koda7 , Xianluo $\mathrm{Ma}^{8}$ and Hong Sun ${ }^{1,3 *}$

${ }^{1}$ Department of Physiology, Xuzhou Medical University, Xuzhou, China, ${ }^{2}$ The College of Biology and Food, Shangqiu Normal University, Shangqiu, China, ${ }^{3}$ Xuzhou Medical University, Xuzhou, China, ${ }^{4}$ Key Laboratory of Bone Marrow Stem Cell, Department of Hematology, The Affiliated Hospital of Xuzhou Medical University, Xuzhou, China, ${ }^{5}$ The Key Laboratory of Cardiovascular Remodeling and Function Research, The State and Shandong Province Joint Key Laboratory of Translational Cardiovascular Medicine, Chinese Ministry of Education, Department of Cardiology, Chinese National Health Commission and Chinese Academy of Medical Sciences, Qilu Hospital of Shandong University, Jinan, China, ${ }^{6}$ Faculty of Biology, Institute of Biochemistry and Molecular Biology, ZBMZ, Albert-Ludwigs University of Freiburg, Freiburg, Germany, ${ }^{7}$ Laboratory of Infection and Immunity, Department of Pathogenic Biology and Immunology, Xuzhou Medical University, Xuzhou, China, ${ }^{8}$ Internal Medicine-Cardiovascular Department, People's Hospital of Jiawang District, Xuzhou, China

Chronic catecholamine stress (CCS) induces the occurrence of cardiomyopathy pathological cardiac hypertrophy $(\mathrm{PCH})$, which is characterized by left ventricular systolic dysfunction (LVSD). Recently, mounting evidence has implicated myocardial inflammation in the exacerbation of pathological cardiac remodeling. However, there are currently no well-defined treatment interventions or regimes targeted at both the attenuation of maladaptive myocardial hypertrophy and inflammation during CCS to prevent $\mathrm{PCH}$. G protein-coupled receptor kinase 5 (GRK5) and adenylyl cyclases (ACs)-CAMP mediates both cardiac and inflammatory responses. Also, GRK5 and ACs are implicated in stress-induced LVSD. Herein, we aimed at preventing PCH during CCS via modulating adaptive cardiac and inflammatory responses by inhibiting GRK5 and/or stimulating ACs. Isoproterenol-induced cardiomyopathy (ICM) was modeled using $0.5 \mathrm{mg} / 100 \mathrm{~g} /$ day isoproterenol injections for 40 days. Alterations in cardiac and inflammatory responses were assessed from the myocardia. Similarities in the immunogenicity of cardiac troponin I (cTnl) and lipopolysaccharide under CCS were assessed, and Amlexanox (35 $\mu \mathrm{M} / \mathrm{ml}$ ) and/or Forskolin (10 $\mu \mathrm{M} / \mathrm{ml})$ were then employed in vitro to modulate adaptive inflammatory responses by inhibiting GRK5 or activating ACs-cAMP, respectively. Subsequently, Amlexanox ( $2.5 \mathrm{mg} / 100 \mathrm{~g} /$ day) and/or Forskolin $(0.5 \mathrm{mg} / 100 \mathrm{~g} /$ day) were then translated into in vivo during CCS to modulate adaptive cardiac and inflammatory responses. The effects of Amlexanox and Forskolin on regulating myocardial systolic functions and inflammatory responses during CCS were ascertained afterward. $\mathrm{PCH}$ mice had excessive myocardial hypertrophy, fibrosis, 
and aggravated LVSD, which were accompanied by massive $\mathrm{CD}^{+} 8^{+}$inflammatory cell infiltrations. In vitro, Forskolin-AC/cAMP was effective than Amlexanox-GRK5 at downregulating proinflammatory responses during stress; nonetheless, Amlexanox and Forskolin combination demonstrated the most efficacy in modulating adaptive inflammatory responses. Individually, the translated Amlexanox and Forskolin treatment interventions were ineffective at subduing the pathological remodeling and sustaining cardiac function during CCS. However, their combination was potent at preventing LVSD during CCS by attenuating maladaptive myocardial hypertrophy, fibrosis, and inflammatory responses. The treatment intervention attained its potency mainly via Forskolin-ACs/CAMP-mediated modulation of cardiac and inflammatory responses, coupled with Amlexanox inhibition of GRK5 mediated maladaptive cascades. Taken together, our findings highlight the Amlexanox and Forskolin combination as a potential therapeutic intervention for preventing the occurrence of pathological cardiac hypertrophy during chronic stress.

Keywords: chronic catecholamine stress, amlexanox, forskolin, GRK5, adenylyl cyclase, cAMP, inflammation, isoproterenol-induced cardiomyopathy

\section{BACKGROUND}

The elevated level of circulating catecholamines during chronic stress is a hallmark for the initiation of adverse remodeling of the left ventricular (LV) (Frey et al., 2004; Liu et al., 2015; Zhang et al., 2019). The progression in LV remodeling has been demonstrated to result in irreversible pathological cardiac hypertrophy $(\mathrm{PCH})$ if there are no timely preventive measures to subdue the excessive firing of neurohormonal stimuli during chronic stress (Schaeuble et al., 2019; Zhang et al., 2019). In recent studies, irreversible hypertrophic cardiomyopathy has been associated with LV systolic dysfunction (LVSD) (Marstrand et al., 2020). Also, the resulting LVSD has been attributed to the distortion of the typical myocardial architecture due to increased LV wall thickness and fibrosis (Galati et al., 2016; Marstrand et al., 2020), thereby making the heart incapable of rapidly replenishing blood for the subsequent ejections (Xie et al., 2013). Although the mechanisms underlying the cardiomyopathy are still being elucidated, the most recent findings have implicated hyperactive myocardial proinflammation responses as a factor hastening the pathological remodeling (Zhao et al., 2017; Weisheit et al., 2021).

In steady state, cardiac resident macrophages have been shown to play crucial roles in reparative functions after cardiac injuries (Heidt et al., 2014). However, an enormous amount of cardiomyocyte deaths (via both apoptosis and necrosis) occur during chronic catecholamine stress (CCS) (Whelan et al., 2010; Liu et al., 2015). The apoptotic myocyte directly induces fibrosis (Liu et al., 2015), while troponins released from the necrotic cardiomyocytes serve as a damage-associated

Abbreviations: AC, adenylyl cyclase; ALX, Amlexanox; cAMP, cyclic adenosine monophosphate; CCS, chronic catecholamine stress; cTnI, cardiac troponin I; FSK, Forskolin; GRK, G protein-coupled receptor kinases; ISO, isoproterenol; LPS, lipopolysaccharide; LVSD, left ventricular systolic dysfunction; PCH, pathological cardiac hypertrophy; $\mathrm{PM}_{\phi}$, peritoneal macrophage; TBK1, TANK-binding kinase 1; TFs, transcriptional factors; TNF $\alpha$, tumor necrosis factor-alpha; $\beta \mathrm{AR}$, betaadrenergic receptors. molecular pattern that invokes proinflammatory responses from macrophages in an attempt to curb further deaths (Haider and Stimson, 1993; Kaya et al., 2008; Heidt et al., 2014). However, under CCS conditions, prolonged secretion of proinflammatory cytokines; interleukin (IL) $1 \beta$, IL-2, IL6 , tumor necrosis factor-alpha (TNF $\alpha)$, and interferon-gamma $(\mathrm{IFN}-\gamma)$ results, while the anti-inflammatory cytokines; IL-10, and transforming growth factor-beta (TGF- $\beta$ ) secretions are downregulated (Kaya et al., 2010). The upregulated secretions of IL- 6 and TNF $\alpha$ prolong myofibroblast activations and increases interstitial fibrosis markedly, which stiffens the myocardia and causes LVSD (Kaya et al., 2010; Hartupee and Mann, 2016; Hulsmans et al., 2016).

Elucidation of the pathomechanisms underlying the occurrence of PCH has shown the involvements of $\beta$-adrenergic receptors ( $\beta$ ARs) and $G$ protein-coupled receptor kinases 5 (GRK5) activities in both cardiomyocytes and immune cells (Hullmann et al., 2014; Adzika et al., 2019). Stimulation of $\beta$ ARs by physiological levels of catecholamines inducing signaling via the stimulatory $G$ protein $\left(G_{\alpha s}\right)$-adenylyl cyclases (ACs) - cyclic adenosine monophosphate (cAMP) pathway (Adzika et al., 2019). In cardiomyocytes, AC5/AC6-cAMP-L-type calcium channel (LTCC) facilitates inotropic and chronotropic, while in immune cells, AC7-cAMP exerts adaptive immunoregulation on transcriptional factors (TFs) as such; nuclear factor of activated T-cells (NFATs), and NF-kB (Paur et al., 2012; Raker et al., 2016; Murphy et al., 2019). As such, ACs-cAMP activities are essential for sustaining the myocardial function under physiological states. However, during CCS, signaling via the $\mathrm{G}_{\alpha \mathrm{s}} / \mathrm{ACs} / \mathrm{cAMP}$ pathway is impeded, affecting its modulation of cardiac and immune functions.

GRKs modulate the expression and activities of $\beta$ ARs. While GRK2 engages in $\beta A R s$ desensitizes, GRK5 phosphorylates the receptors to initiate $G$ protein-coupled receptors (GPCR)independent signaling, which induces PCH (Gold et al., 2012; Hullmann et al., 2014; Traynham et al., 2016). Also, GRK5 
translocations into nuclei to maladaptively phosphorylate cardiac hypertrophy and inflammatory TFs; NFATs, myocyte enhancer factor 2 (MEF2), GATA4, and NF-kB hastens the pathological remodeling (Hullmann et al., 2014).

Currently, there are no stipulated treatment regimens that aim at attenuating PCH during CCS through the simultaneous prevention of maladaptive myocardial hypertrophy, fibrosis, and inflammation. Herein, we demonstrate that during chronic isoproterenol-induced cardiomyopathy (referred to as $\mathrm{PCH}$ hereafter), the expression of $\beta$ ARs, ACs, GRKs, cardiac hypertrophy, and inflammatory TFs are altered. Also, we showed that inflammatory responses elicited by troponin in the $\mathrm{PCH}$ hearts were similar to those induced when its activity was mimicked in vitro with lipopolysaccharides (LPS) during stress.

GRK5 and ACs were targeted with amlexanox (ALX) and forskolin (FSK), respectively, to explore $\mathrm{PCH}$ treatment interventions. ALX has been shown to inhibit GRK5-mediated hypertrophy in PCH models; however, there is no evidence that cardiac functions were preserved (Homan et al., 2014). Also, ALX is demonstrated to inhibit inflammatory responses activated via GRK5 (Patial et al., 2011; Quan et al., 2019). The stimulation of ACs by FSK facilitates the bioavailability of cAMP (Huang and Wong, 1989; Chiadak et al., 2016), which might ensure the modulation of cardiac and inflammatory functions during CCS. Therefore, by inhibiting GRK5 and stimulating ACs, we attenuated the hyperactive proinflammatory responses observed in vitro. Furthermore, these treatment interventions were translated in vivo to assess their therapeutic potential in attenuating $\mathrm{PCH}$ through modulation inflammatory and hypertrophy responses.

\section{MATERIALS AND METHODS}

\section{Experimental Animals and Models}

Male FVB mice aged between 8 and 12 weeks were randomized into groups ( $n=4-16$ mice per group depending on the experimental setting) and used for both in vitro and in vivo experiments. Isoproterenol-induced cardiomyopathy models were made by subcutaneous injections of isoproterenol (160504; Sigma) $0.5 \mathrm{mg} / 100 \mathrm{~g} /$ day as previously demonstrated (Lin et al., 2016; Zhou et al., 2017), for 40 days. The equivalents of the placebo (5\% v/v dimethyl sulfoxide) were injected to the vehicle (Vhl) group. Also, because it has been shown that laboratory animal handling induces background stress (Gouveia and Hurst, 2019), littermates without any handling were included in the study and designated as the control (Ctrl) group to rule out handling as a contributor to any observed pathological alterations.

$\mathrm{PCH}$ preventive models were made by intraperitoneal injection of ALX (ab142825; Abcam) $2.5 \mathrm{mg} / 100 \mathrm{~g} /$ day and/or FSK (1099; Tocris Bioscience, United Kingdom) $0.5 \mathrm{mg} / 100 \mathrm{~g} /$ day. These treatments were administered during the isoproterenol-induced cardiomyopathy modeling (Supplementary Figure 1).

The mice were euthanatized by cervical dislocation, and macrophages or the hearts were isolated for further analysis.

\section{Electrocardiography and Echocardiography}

Mice, 10-11 per treatment group, were mildly sedated with $0.5 \%$ isoflurane. The mice were fixed with echo gel, and their body temperatures were monitored and maintained at $37^{\circ} \mathrm{C}$ for electrocardiography (ECG) data acquisition with Vevo 2100 Ultrasound system (VisualSonics, Toronto, Canada). Simultaneously, global cardiac function was assessed in M-mode with a Vevo 2100 Ultrasound system (VisualSonics, Toronto, Canada). At end-systole and end-diastole, the interventricular septal wall and posterior wall thicknesses, as well as left ventricle internal diameters, were evaluated from the left parasternal longitudinal axis view. Ejection fraction (EF) and fractional shorten (FS) were calculated as described (Jia et al., 2019).

\section{Immune Cell Isolation and Culture}

Peritoneal macrophages $\left(\mathrm{PM}_{\phi}\right)\left(n \geq 2 * 10^{6}\right.$ cells per treatment group) were isolated from mice as previously described (Ray and Dittel, 2010) but with some modifications. Eight to ten milliliters of $37^{\circ} \mathrm{C}$ PBS containing $3 \%$ fetal bovine serum (FBS) was carefully injected into the peritoneal cavity. The cell suspensions were then collected from the peritoneum after $5 \mathrm{~min}$ and centrifuged at $1,500 \mathrm{rpm}$ for $10 \mathrm{~min}$. The pellets of cells were resuspended in $10 \%$ FBS and cultured for $48 \mathrm{~h}$. The cells were initially identified using F4/80 (123116; BioLegend) and CD11b (101206; BioLegend). Subsequently, cultured $\mathrm{PM}_{\phi}$ were pre-treated for $1 \mathrm{~h}$ with $35 \mu \mathrm{M} / \mathrm{ml}$ of ALX, and challenged with $10 \mu \mathrm{M} / \mathrm{ml}$ of ISO and/or $1 \mu \mathrm{g} / \mathrm{ml}$ LPS along with the same dose of ALX treatment for $24 \mathrm{~h}$. Also, $\mathrm{PM}_{\phi}$ challenged with ISO and LPS was treated with $10 \mu \mathrm{M} / \mathrm{ml} \mathrm{FSK}$. The combination of ALX and FSK treatment was employed as well. The supernatants were used for ELISA assays, while the $\mathrm{PM}_{\phi}$ were used to assess GRK5 activities in vitro.

\section{Enzyme-Linked Immunosorbent Assay}

Sera and lysate from in vivo models $(n=7-9$ mice per treatment group) and cell culture media supernatants from treated $\mathrm{PM}_{\phi}$ were used to assess the concentrations of proinflammatory cytokines (IL-1 $\beta$, IL-6, and TNF $\alpha$ ), anti-inflammatory cytokines (IL-10 and TGF- $\beta$ ), troponin I and cAMP. IL-1 $\beta$ (ab197742; Abcam), IL-6 (ab222503; Abcam), TNF $\alpha$ (ab208348; Abcam), IL10 (ab255729; Abcam), TGF- $\beta$ (KE10005; Proteintech), cAMP (JL13362; Jianglai Bio, China), and troponin I (JL31923; Jianglai Bio, China) ELISA were performed in triplicates as per the instructions of the manufacturer.

\section{RNA Extraction and Real-Time qPCR}

Total RNA was extracted from ventricular apical myocardium ( $n=6$ hearts per treatment group) using TRIzol (15596026; Life technologies). cDNA was synthesized from $1 \mu \mathrm{g}$ of RNA using a reverse transcription kit according to the instruction of the manufacturer (FSQ107; Toyobo). The obtained cDNAs were amplified by semiquantitative RT-PCR using SYBR Green Master Mix (Q111-02; Vazyme). The primer sequences used are listed here (Supplementary Table 1). The relative gene expressions were normalized to the expressions of GAPDH and were compared as described (Gold et al., 2012). 


\section{Immunofluorescence Staining}

Cultured and pre-treated $\mathrm{PM}_{\phi}\left(n \geq 2 * 10^{6}\right.$ cells per treatment group) were fixed and permeabilized with pre-chilled methanolacetone (ratio 1:1). Non-specific antibody binds were blocked with $1 \%$ BSA in PBS for $1 \mathrm{~h} . \mathrm{PM}_{\phi}$ were then incubated with GRK5 primary antibody (ab64943; Abcam) at $4^{\circ} \mathrm{C}$ overnight, washed with PBS, and probed with R-PE-conjugated secondary antibody (SA00008-2; Proteintech) at room temperature for $1 \mathrm{~h}$. Next, cells were washed with PBS, conditioned with $0.5 \%$ BSA in Hanks' balanced salt solution, and the cytoplasmic membranes were stained with cholera toxin B (CTxB) (C34775, Thermo Fisher Scientific) for $30 \mathrm{~min}$ at $4^{\circ} \mathrm{C}$. DAPI nuclei staining were done and followed by imaging and assessment of the ratios of nucleic and cytoplasmic GRK5 expressions with ImageJ ( $n=12-15$ cells per four mice).

\section{Histological Analysis of Hypertrophy, Interstitial Fibrosis, and Immune Cells Infiltration}

Hearts were excised ( $n=6-10$ mice per treatment group), rinsed, and fixed in $10 \%$ formaldehyde overnight. The hearts were then embedded in paraffin and sectioned at $4 \mu \mathrm{m}$. Subsequently, H\&E (G1120; Solarbio), Masson's trichrome (G1340; Solarbio), wheat germ agglutinin (WGA) (W11261; Thermo Fisher Scientific), and CD68 (ab955; Abcam) immunohistochemical staining were then performed as previously described (Qi et al., 2019). Imaging of tissue slides was done at $\times 400$ magnification and analyzed with ImageJ (1.52a version; National Institute of Health, United States).

\section{Western Blot}

Apical myocardia harvested from four to six hearts per treatment group were homogenized in a lysis buffer containing phosphatase and proteinase cocktail inhibitor, in a ratio of 100:1:1, respectively. Lysates of normalized concentrations treated with reducing agents were denatured at $100^{\circ} \mathrm{C}$ for $10 \mathrm{~min}$ and separated on SDS-PAGE gels. The transferred protein bands were blocked and immunoblotted overnight in the following primary antibodies: $\beta_{1} \mathrm{AR}$ (ab3442; Abcam), $\beta_{2}$ AR (ab182136; Abcam), MEF2 (ab64644; Abcam), GRK5 (ab64943; Abcam) GATA4 (ab84593; Abcam), NFAT (ab25916; Abcam), AC5 (PAC-501AP; FabGennix), AC6 (PAC-601AP; FabGennix), AC7 (PAC-701AP; FabGennix), ANP (sc-515701; Santa Cruz Biotechnology), BNP (sc-271185; Santa Cruz Biotechnology), GRK2 (sc-13143; Santa Cruz Biotechnology), pNF-кB (3033T; Cell Signaling Technology), NF-кB (8242T; Cell Signaling Technology), Cleaved Caspase-3 (9661T; Cell Signaling Technology), Collagen Type I (14695-1-AP, Proteintech), Collagen Type III (13548-1-AP, Proteintech), and GAPDH (10494-1-AP; Proteintech). Immunoblots were performed in triplicates and normalized with their respective loading controls.

\section{Statistical Analysis}

One-way ANOVA was used for comparing data of multiple groups, and post hoc analyses were done using Tukey's multiple comparisons test. Values of $p<0.05$ were deemed significant.
Using GraphPad Prism (Prism Version 8.0.2), all data were expressed as mean $\pm \mathrm{SEM}$.

\section{RESULTS}

\section{Chronic Catecholamine Stress Alters Cardiac and Inflammatory Functional Proteins in Mice}

Analysis of mRNAs and protein expressions demonstrated that crucial cardiac and inflammatory proteins were altered in the myocardia during CCS compared with under physiological states. $\beta A R s, A C 5$, and AC7 were found depleting, while AC6, $\beta$-ARR- 1 , $\beta$-ARR-2, GRK2, and GRK5 were upregulated in the PCH mice hearts (Supplementary Figures 2A-K). Also, the TFs: GATA4, NFAT, MEF2, and NF-KB were overexpressed in PCH hearts compared with Ctrl and Vhl hearts (Supplementary Figures 3AE). Cardiac hypertrophy was evident in $\mathrm{PCH}$ mice as atrial natriuretic peptide (ANP) and brain natriuretic peptide (BNP) were significantly upregulated in their myocardia than Ctrl and Vhl mice (Supplementary Figures 2A,J,K). To ascertain the impact of AC5, AC6, and AC7 alterations in $\mathrm{PCH}$ models, cAMP bioavailability was assessed and found to be significantly decreased in myocardial lysates obtained from $\mathrm{PCH}$ mice (Supplementary Figure 2L).

\section{Troponin and Lipopolysaccharide Elicit Similar Immunogenic Response During Chronic Catecholamine Stress}

Immediate assays for cardiac troponin I (cTnI) from sera showed its overt upregulation in $\mathrm{PCH}$ mice compared with concentrations in Ctrl and Vhl mice (Figure 1A). Assessment of mRNAs of IL-1 $\beta$, IL-6, TNF $\alpha$, IFN $\gamma$, NF- $\kappa B$, and IL-10 from the myocardia showed that besides the latter, the proinflammatory cytokines and TF were significant overexpression in $\mathrm{PCH}$ hearts (Figure 1B).

By using LPS to mimic the immunogenicity of troponin in vitro, inflammatory responses elicited under stress were ascertained and compared with the in vivo models. The result demonstrated similarities in their immunogenicity. The $\mathrm{PM}_{\phi}$ culture with ISO and LPS for $24 \mathrm{~h}$ secreted enormous amounts of IL-1 $\beta$, IL-6, and TNF $\alpha$ just as observed in PCH mice, while IL10 and TGF- $\beta$ were also dampened similarly, both in vitro and in vivo (Figures 1C-G).

\section{Amlexanox and Forskolin Combination Inhibits Proinflammatory Responses During Chronic Catecholamine Stress Primarily via Cyclic Adenosine Monophosphate-Mediated Immunoregulation}

ALX $(35 \mu \mathrm{M} / \mathrm{ml})$ and/or FSK $(10 \mu \mathrm{M} / \mathrm{ml})$ treatments were employed to attenuate the hyperactive proinflammatory cytokine secretions elicited from the $\mathrm{PM}_{\phi}$ in vitro. Analysis of the concentrations of inflammatory cytokines from these 

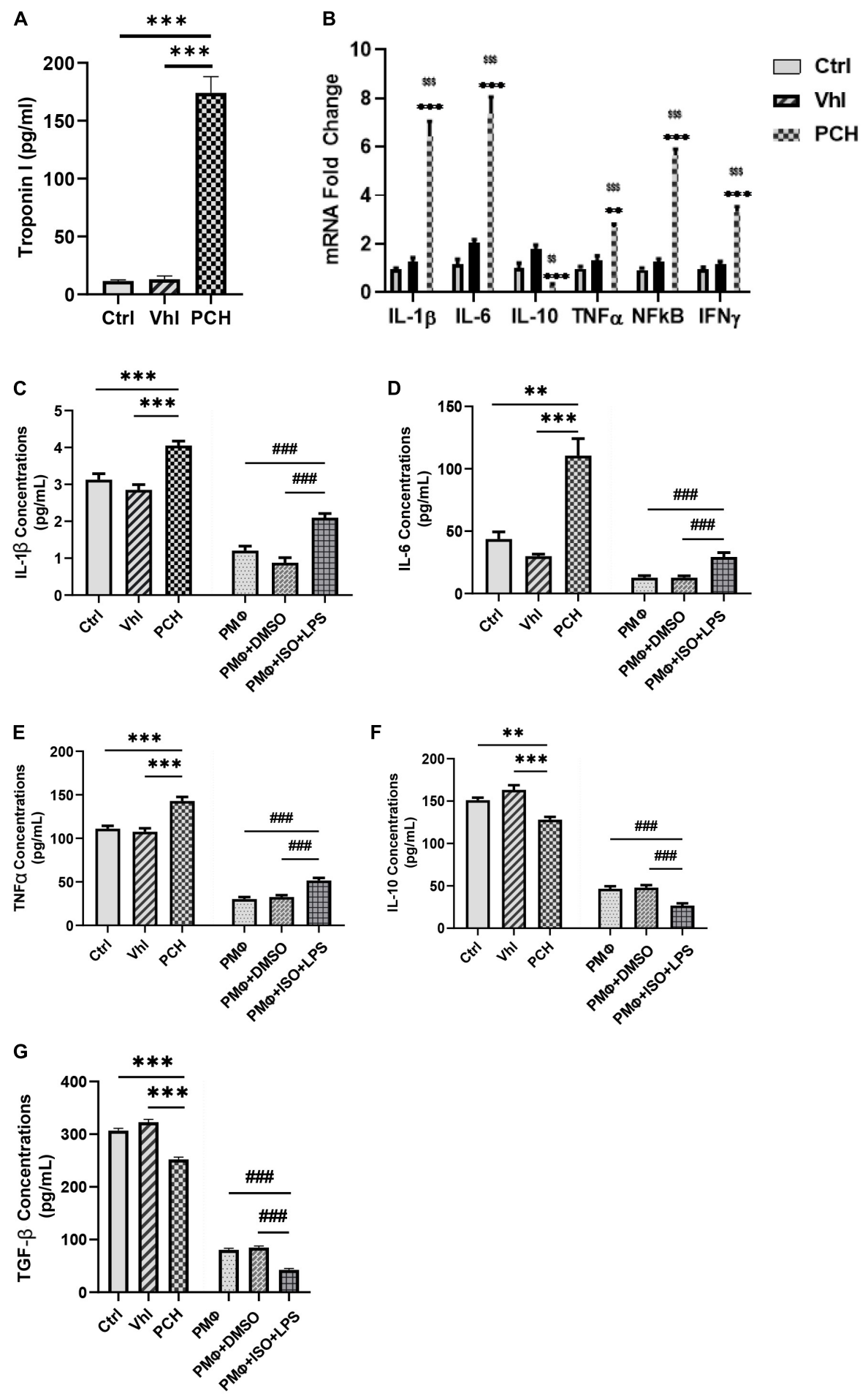

FIGURE 1 | Troponin from necrotic cardiomyocytes and lipopolysaccharides (LPS) elicits similar inflammatory responses during chronic catecholamine stress (CCS). (A) Sera concentrations of troponin I were evaluated by ELISA from Control (Ctrl), Vehicle (Vhl), and Pathological cardiac hypertrophy (PCH) groups. *** $p<0.001$ (B) mRNA expressions of inflammatory markers (IL-1 $\beta, \mathrm{IL}-6, \mathrm{TNF} \alpha$, IFN $\gamma$, and NF-kB) assessed from the myocardial by qRT-PCR ( $n=6$ hearts per treatment group). ${ }^{* *} p<0.01,{ }^{* * *} p<0.001$ vs. Vhl; ${ }^{\$} p<0.01, \$ \$ \$ p<0.001$ vs. Ctrl. (C-G) Comparison with inflammatory cytokines secretion (IL-1 $1 \beta$, IL-6, TNF $\alpha$, IL-10, and TGF- $\beta$ ) between $\mathrm{PCH}$ mice (in vivo) and LPS-challenged peritoneal macrophages (PM $\phi$ ) (in vitro) during CCS. All ELISA evaluations were performed in triplicates $(n=8$ mice per treatment group). ${ }^{* *} p<0.01,{ }^{* * *} p<0.001$ among in vivo groups; $\# \#$ \#\# 0.001 among in vitro groups. Data are expressed as mean $\pm \mathrm{SEM}$. Data were analyzed using one-way ANOVA, followed by Tukey's post hoc analysis. Abbreviations: IL, interleukin; TNFa, tumor necrosis factor-alpha; TGF- $\beta$, transforming growth factor-beta; IFNy, interferon-gamma. 
experiments using ELISA demonstrated that ALX treatment could not effectively subdue the hypersecretions of IL- $1 \beta$, IL- 6 , and TNF $\alpha$; neither did it upregulate IL-10 and TGF- $\beta$ secretions during stress. Comparatively, FSK treatment was effective than ALX treatment at decreasing the proinflammatory responses while upregulating IL-10 and TGF- $\beta$ secretions (Figures 2A-E). Regardless, ALX and FSK combination treatment was the most potent in subduing the hyperactive proinflammatory response.

Assessment of the effect of FSK on ensuring cAMP bioavailability showed that its presence in both the single and combination treatments upregulated cAMP markedly compared with the ISO + LPS and ISO + LPS + ALX groups (Figure 2F). Hence, it is inferred that the potency attained by the combination treatment may have been mainly via cAMPmediated immunoregulation. The validation of this was done by assessing the effect of the therapies on GRK5 expressions. GRK5 cytosolic and nuclear expressions were inhibited by ALX in both the single and combination treatments, while FSK treatment had increased GRK5 nucleic-cytosolic expression ratio just as ISO + LPS, which had no treatment interventions (Figures 2G,H). However, proinflammatory responses were minimized in the ISO + LPS + FSK (Figures 2A-C), despite the increase in GRK5 nuclear expression.

\section{Amlexanox and Forskolin Combination Attenuates Left Ventricular Systolic Dysfunction in Mice During Chronic Catecholamine Stress}

Echocardiography and ECG assessments at the end of in vivo model showed that PCH mice had LVSD. The treatments of ALX, FSK, and their combination were employed during CCS in attempts to prevent the LVSD. Cardiac functions assessed after using these treatment interventions showed that ALX failed to prevent the LVSD during CCS, as ejection fraction (EF) and fractional shortening (FS) were still decreased significantly
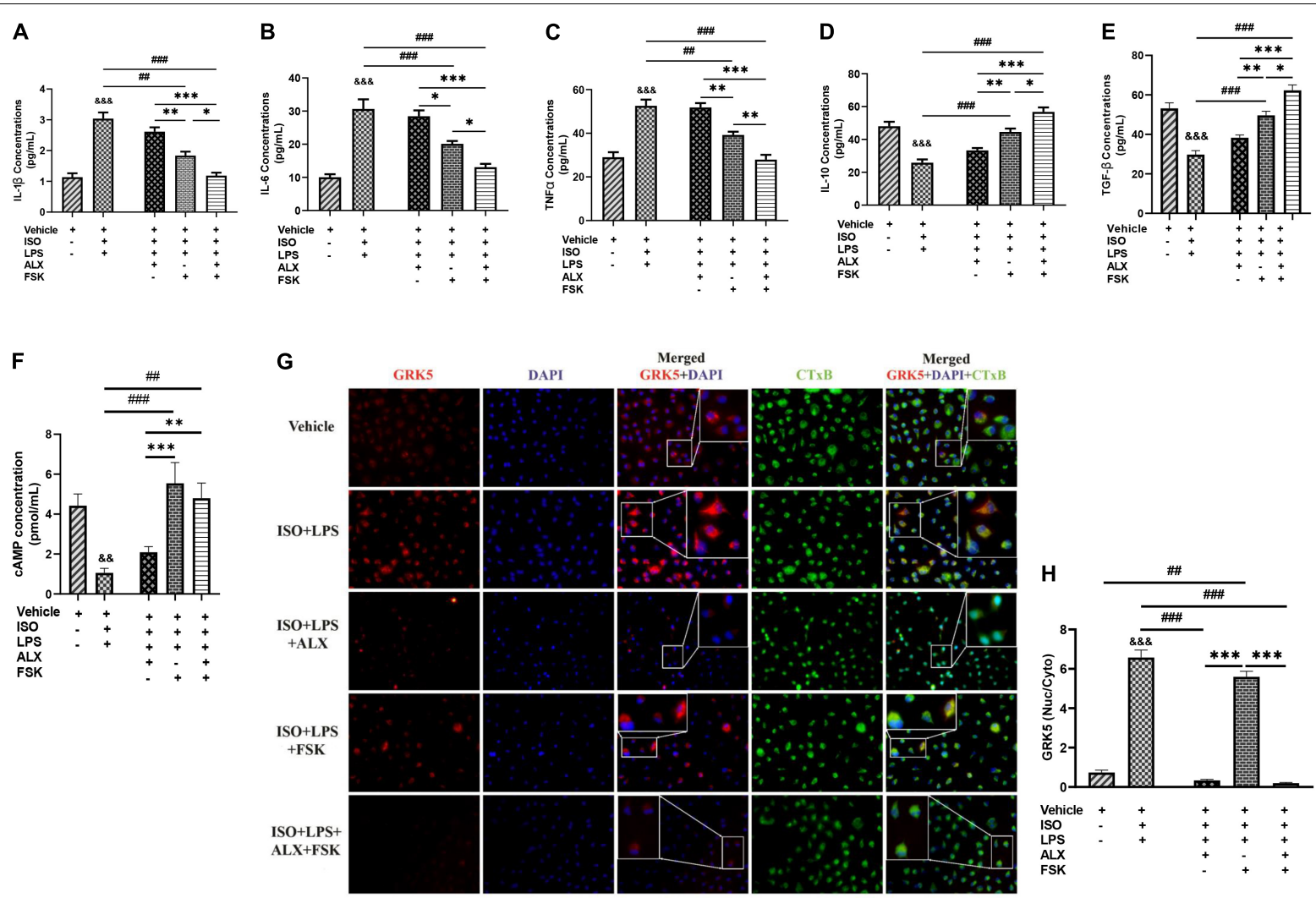

FIGURE 2 | Amlexanox (ALX) and forskolin (FSK) combination inhibits $\mathrm{PM}_{\phi}$ proinflammatory responses elicited during CCS, mostly via cyclic adenosine monophosphate (cAMP)-mediated immunoregulation. (A-E) ELISA evaluation of inflammatory cytokines (IL-1 $\beta$, IL-6, TNF $\alpha$, IL-10, and TGF- $\beta$ ) secreted by LPS-challenged $\mathrm{PM}_{\phi}$ after employing ALX and/or FSK treatment intervention during CCS. (F) CAMP concentrations were assessed to determine the impact of the treatment interventions on ensuring CAMP bioavailability during CCS. The therapeutic groups are ALX treatment, FSK treatment, and ALX and FSK combination treatment. All ELISA evaluations were performed in triplicates ( $n=8$ mice per treatment group). ${ }^{*} p<0.05,{ }^{* *} p<0.01,{ }^{* * *} p<0.001$ among the therapeutic groups; $\# p<0.01, \# \# p<0.001$ LPS $+{ }^{\# M_{\phi}}+$ ISO vs. therapeutic groups; ${ }^{\& \&} p<0.01,{ }^{\& \& \&} p<0.001$ vs. LPS $+\mathrm{PM}_{\phi}+$ Vhl. (G) Representative immunofluorescence of G protein-coupled receptor kinase 5 (GRK5) localizations, nuclei (DAPI), and cytoplasmic membrane (CTXB). (H) The plotted values are the GRK5 (nuclear/cytoplasm) expression ratios assessed from each $\mathrm{PM}_{\phi}(n=12-15$ cells per four mice per treatment group). Color channels were adjusted in the merged images to enhance the visualization of all the respective fluorescence dyes. ${ }^{* * *} p<0.001$ among the therapeutic groups; $\#$ $p<0.01, \# \# p<0.001$, LPS + PM ${ }_{\phi}+$ ISO vs. therapeutic groups; ${ }^{\& \&} \&<0.001$ vs. LPS $+\mathrm{PM}_{\phi}+$ ISO. Data are expressed as mean \pm SEM. Data were analyzed using one-way ANOVA, followed by Tukey's post hoc analysis. 
compared with the control groups. Similarly, FSK treatment did not attenuate the LVSD during CCS but instead caused tachycardia and arrhythmias (Figures 3A-D and Supplementary Figure 3F and Supplementary Table 2). Intriguingly, ALX and FSK combination was found to preserve cardiac function by maintaining EF and FS during CCS.

\section{Amlexanox and Forskolin Combination Normalizes Cardiac and Inflammatory Functional Protein Expression in Mice During Chronic Catecholamine Stress}

To ascertain the potential mechanism of action employed by the ALX and FSK combination to prevent the LVSD, the expression of functional proteins and TFs were reassessed. $\beta$ ARs, AC5, AC6, AC7, GRK2, GRK5, ANP, BNP, GATA4, NFAT, MEF2, and NF- $\kappa$ B expressions were found to have been altered in PCH mice myocardia (Supplementary Figures 2, 3). However, the ALX and FSK combination prevented significant alteration in the expressions of these proteins, while their individual treatment showed several limitations. $\beta_{1} \mathrm{AR}$ and $\beta_{2} \mathrm{AR}$ expressions were sustained by all treatment intervention groups.
FSK in both single and combination treatment normalized AC5 and AC7 to physiological levels, while AC6 was upregulated; thus, cAMP concentrations were primarily increased in these groups. Meanwhile, ALX showed a negligible inhibitory effect on GRK2 but prevented GRK5, ANP, and BNP upregulations in both its single and combined treatments with FSK (Figures 3E-O). Nonetheless, GATA4, NFAT, MEF2, and NF- $\kappa$ B expressions were still upregulated predominantly in the ALX treatment group and followed by the FSK treatment group. Intriguingly, ALX and FSK combination minimized the expressions of these functional proteins and TFs close to physiological levels during CCS (Supplementary Figures 4A-E).

\section{Amlexanox and Forskolin Combination Preserves Mice Myocardial Architecture During Chronic Catecholamine Stress}

LV posterior wall thickness (LVPW), LV mass (LVM), and heart weight/body weight $(\mathrm{HW} / \mathrm{BW})$ ratio assessments showed their significant increases in $\mathrm{PCH}$ mice compared with the Vhl and Ctrl groups (Figure 4A and Supplementary Table 2). However, during CCS, ALX in both single and combination treatment
A

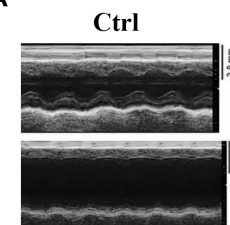

ISO+ALX

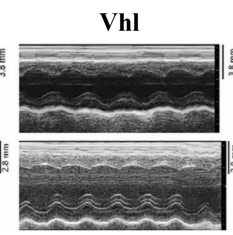

ISO+FSK
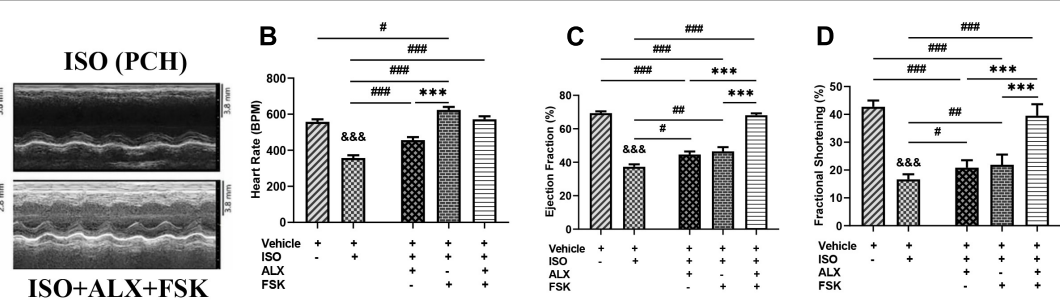

E
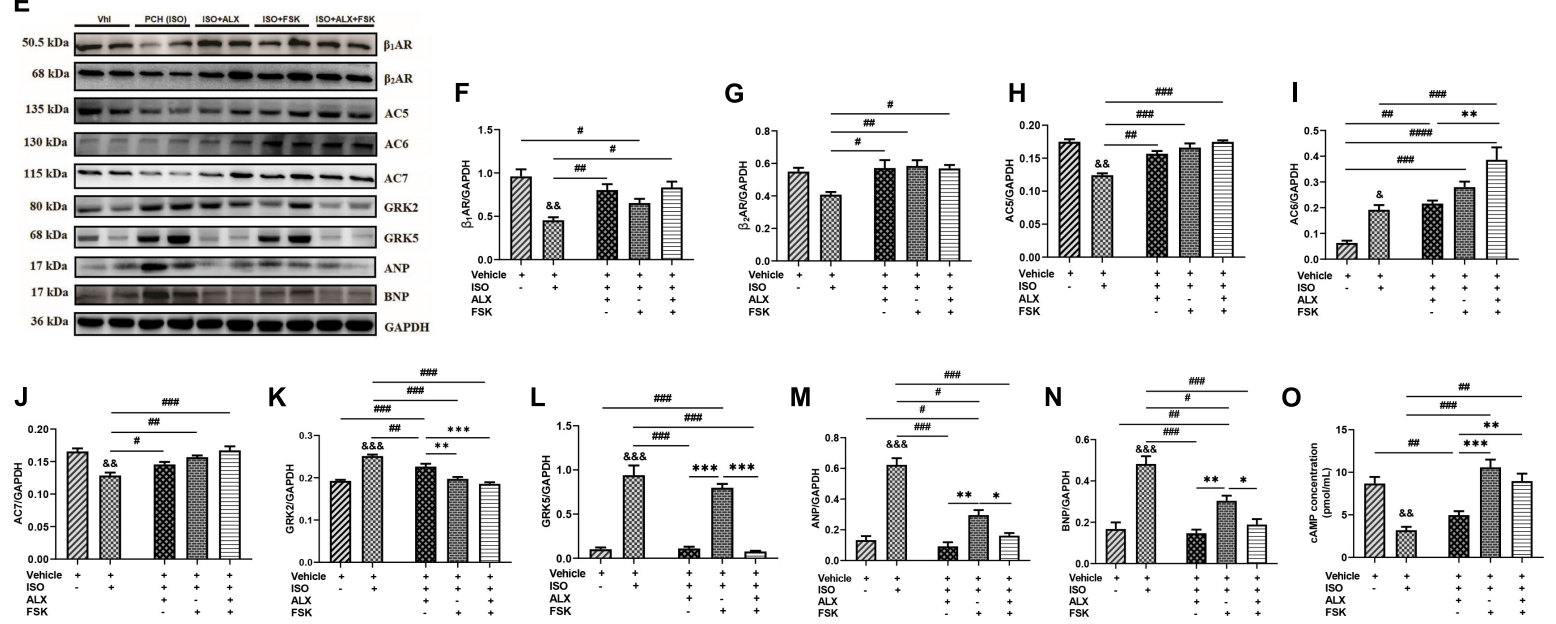

FIGURE 3 | ALX and FSK combination attenuates left ventricular systolic dysfunction (LVSD) in mice during CCS by normalizing protein expressions. (A) Representative short-axis view M-mode echocardiogram imaging from Ctrl, Vhl, isoproterenol (ISO), ISO + ALX, ISO + FSK, and ISO + ALX + FSK. (B-D) Graphical presentations of heart rates, ejection fraction, and fractional shortening assessments from all models. The Vhl mice group is representative of the Ctrl mice group in the graphical presentations due to similarity in their data. The therapeutic groups are ALX treatment, FSK treatment, and ALX and FSK combination treatment ( $n=10-11$ hearts per treatment group). (E-N) Representative Western blots and graphical presentation of $\beta$ ARs, ACs, GRKs, ANP, and BNP, were assessed from all groups ( $n=4$ hearts per treatment group). Western blots were performed in triplicates, and each protein band in the representative blot is an independent biological sample. (0) Graphical represents of cAMP concentrations evaluated by ELISA ( $n=9$ mice per treatment group). ${ }^{*} p<0.05,{ }^{* *} p<0.01$,

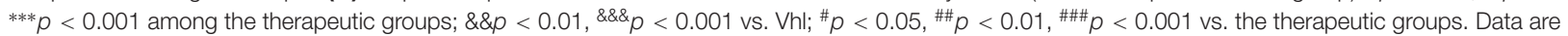
expressed as mean \pm SEM. Data were analyzed using one-way ANOVA, followed by Tukey's post hoc analysis. Abbreviations: $\beta$ ARs, $\beta$-adrenergic receptors; ACs, adenylyl cyclases; GRKs, G-protein-coupled receptor kinases; ANP, atrial natriuretic peptide; BNP, brain natriuretic peptide. 


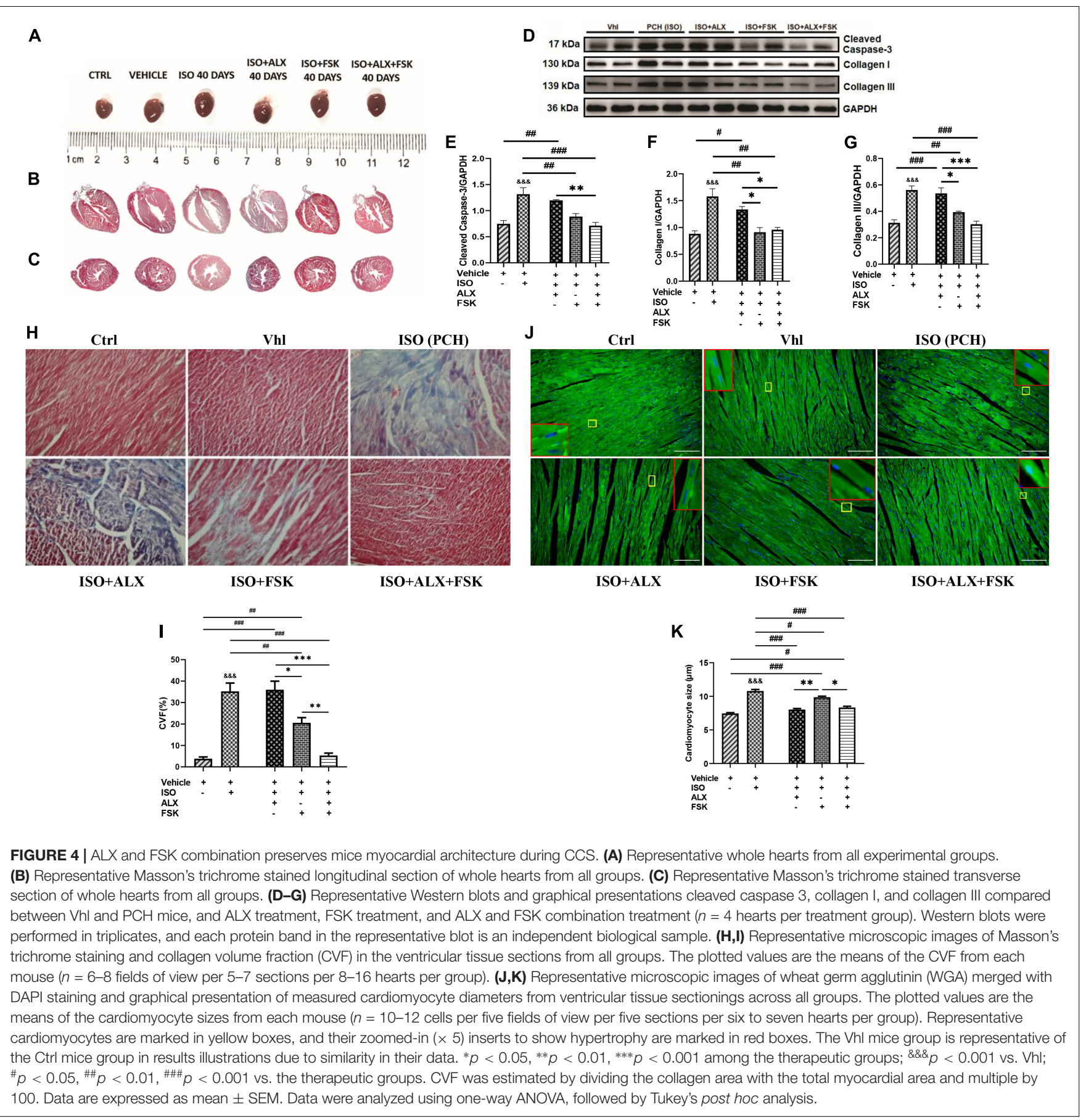

prevented significant increases in LVPW, LVM, and HW/BW; meanwhile, FSK treatment failed to achieve these. Masson's trichrome staining of longitudinal and transverse myocardial sections showed apparent distortions of the gross cardiac morphological of PCH mice. Nonetheless, rather than either ALX or FSK treatments, their combination seemed to have preserved the myocardial architecture during CCS (Figures $\mathbf{4 B}, \mathbf{C}$ ). The impacts of the treatment interventions on cardiomyocyte apoptosis and collagen depositions were ascertained to validate this observation. Cleaved caspase-3 (biomarker for apoptosis) was upregulated in $\mathrm{PCH}$ hearts along with significant increases in collagen I and III. In conformity with the histological findings, only the ALX and FSK combination treatment was potent at preventing cardiomyocyte apoptosis and collagen depositions in the heart of the mice during CCS (Figures 4D-G). Also, the myocardial collagen volume fraction (CVF) assessed further confirmed that PCH mice had marked interstitial deposits, which was only prevented effectively by the ALX and FSK combination treatment (Figures $\mathbf{4 H}, \mathbf{I}$ ). Besides, cardiomyocyte diameters measured from WGA and H\&E staining showed 
excessive myocyte hypertrophy in $\mathrm{PCH}$ hearts. Consistent with attenuating the increases in ANP, BNP, LVPW, HW/BW, and LVM, ALX in both single and combination treatment prevented cardiomyocyte hypertrophy during CCS, while FSK treatment could not (Figures 4J,K and Supplementary Figures 4F,G).

\section{Amlexanox and Forskolin Combination Prevents Proinflammatory Response Aggravation in Mice Myocardia During Chronic Catecholamine Stress}

The effects of the treatment interventions in modulating myocardial inflammation were initially ascertained by evaluating their impact on cardiomyocyte necrosis through the immediate sera analysis of the cTnI. Inference from the cTnI concentrations indicated that the ALX treatment was ineffective at preventing necrosis, while FSK treatment did better comparatively. Regardless, ALX and FSK combination was the most potent in preventing necrosis during CCS (Figure 5A). Furthermore, $\mathrm{CD}^{+} 8^{+}$IHC staining was done to evaluate the efficacies of the treatments in attenuating the aggravated inflammatory cell infiltration observation in $\mathrm{PCH}$ hearts (Figures 5B,C) and demonstrated that, while FSK significantly decreased the $\mathrm{CD}^{+} 8^{+}$cells infiltration than the ALX during CCS, their combination was the most potent at preventing exacerbation of the inflammatory responses. In conformity, the followed-up cytokine analysis showed that the ALX and FSK combination was the most effective in downregulating IL-1 $\beta$, IL-6, and TNF $\alpha$ secretions while enhancing IL-10 and TGF- $\beta$. Comparatively, FSK treatment was able to do these better than ALX treatment did during CCS (Figures 5D-H).

\section{DISCUSSION}

This study aimed to prevent isoproterenol-induced cardiomyopathy through the attenuation of maladaptive myocardial hypertrophy and inflammatory responses. The preliminary assessment of cardiac and inflammatory functional proteins demonstrated their alteration during CCS compared with physiological states. Typically, $\beta_{1} \mathrm{AR}$ expressions were significantly downregulated, while $\beta_{2} \mathrm{AR}$ expressions were rarely depleted during CCS. Consistent with previous studies, $\beta_{2} \mathrm{AR}$ is implicated in mediating stress-induced cardiovascular diseases (Paur et al., 2012; Spadari et al., 2018). Also, while AC5 expression was decreased during CCS, AC6 was found upregulated. In conformity with these findings, it has been previously shown that AC5 facilitates PKA pathway signaling under physiological conditions, while AC6 mediates stress response for calcium channel modulation via PKA/STAT3

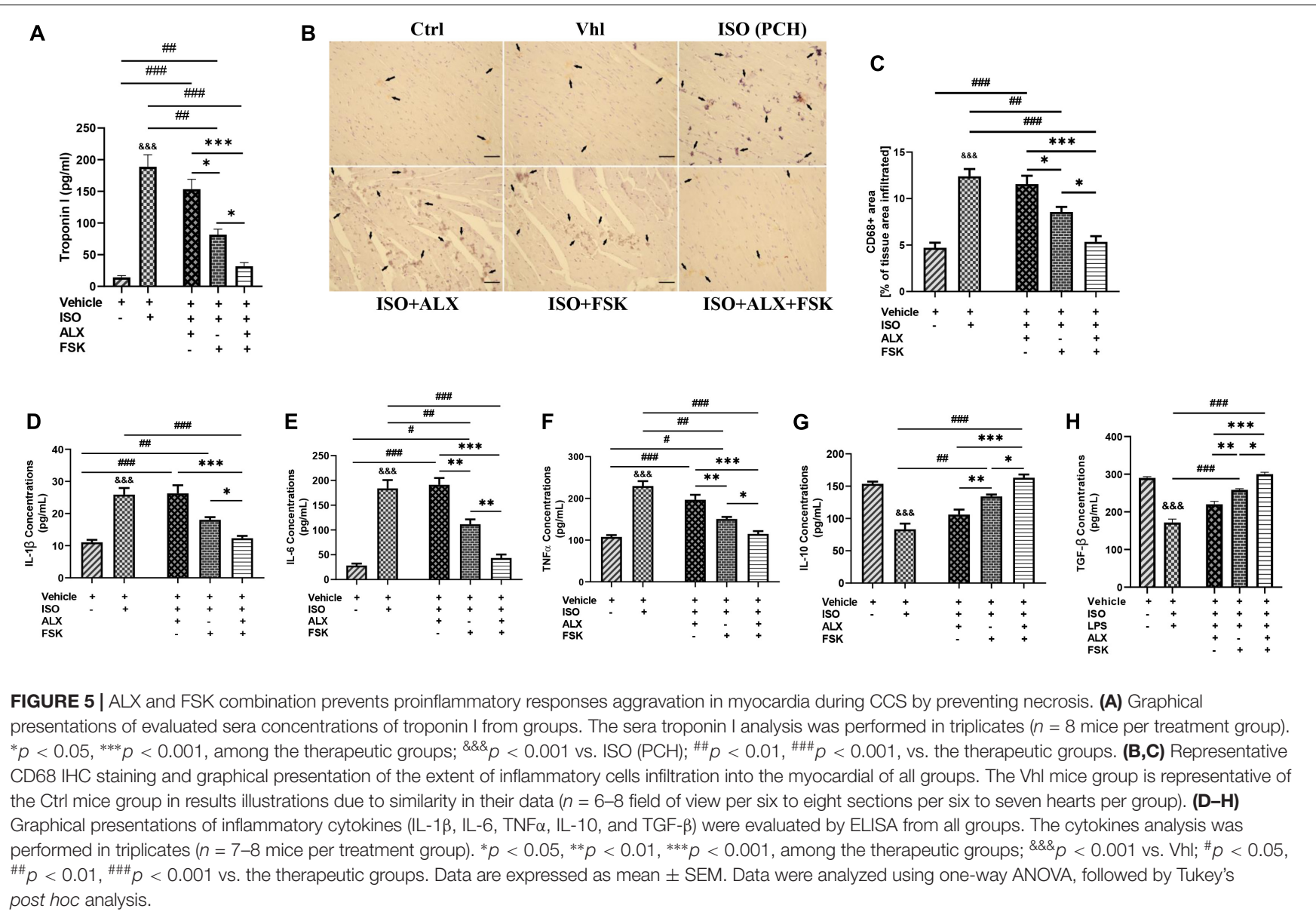


(Wu et al., 2017). Like AC5, AC7 was downregulated in $\mathrm{PCH}$ mice myocardia compared with the Ctrl and Vhl groups. The ELISA, performed to ascertain the impact of the ACs alteration on CAMP bioavailability, showed decreased concentrations in $\mathrm{PCH}$ mice as reported in other chronic stress models (Plattner et al., 2015). cAMP mediates crucial cardiac and immunoregulatory functions via the modulation of the LTCC, NFAT, MEF2, and NF- $\mathrm{B}$ in both inflammatory and cardiac cells (Pereira et al., 2015; Raker et al., 2016). Hence, the observed downregulation of cAMP in $\mathrm{PCH}$ mice might permit cardiac and inflammatory dysfunctions. We also found that $\beta$-ARR-1, $\beta$-ARR-2, GRK2, and GRK5 were overexpressed in $\mathrm{PCH}$ hearts, which were consistent with the findings of others (Hullmann et al., 2014; Adzika et al., 2019; Sun et al., 2021). GRK2 upregulation increases the internalization of $\beta_{1} \mathrm{AR}$ via the recruitment of $\beta$-ARR-1 in the homologous desensitization process (Adzika et al., 2019; Sun et al., 2021); therefore, this explains the downregulated expression of $\beta_{1} \mathrm{AR}$ in $\mathrm{PCH}$ myocardia. Conversely, by recruiting $\beta$-ARR-2, GRK5 enhances $\beta_{2} \mathrm{AR}$ signal progression in a GPCR-independent manner to induce maladaptive hypertrophy and proinflammatory responses via phosphorylating NFAT, MEF2, GATA4, and NF-кB (Patial et al., 2011; Islam et al., 2013; Hullmann et al., 2014; Figure 6). Intriguingly, these cardiac and inflammatory TFs were shown overexpressed, along with ANP and BNP upregulated in the $\mathrm{PCH}$ myocardia compared with their expressions in the control groups (Supplementary Figures 3, 4). These are indicative of the likelihood of GRK5 activities in inflammatory and cardiac cells.

The exacerbation of cardiovascular remodeling by cTnIinduced inflammatory responses has also been widely reported. In these instances, elevated proinflammatory and downregulated anti-inflammatory responses were observed (Kaya et al., 2008; Heidt et al., 2014). The upregulated sera levels of cTnI in $\mathrm{PCH}$ mice (Figure 1A) indicate the occurrence of excessive cardiomyocyte necrosis. In line with the increased release of cTnI by necrotic cardiomyocytes, proinflammatory responses (IL-1 $\beta$, IL-6, TNF $\alpha$, IFN $\gamma$, and NF- $\kappa \mathrm{B}$ ) were hyperactive in $\mathrm{PCH}$ mice, while anti-inflammatory responses (IL-10 and TGF- $\beta$ ) were dampened, just as demonstrated previously (Kaya et al., 2008).

Furthermore, LPS has been extensively used to induce cardiac inflammation and has been observed to share similarities in the immunogenic responses elicited by troponin (Cai et al., 2020). To validate this, we mimicked cTnI immunogenicity in PCH models with LPS under stress conditions in vitro. Comparing cytokine analysis between in vivo and in vitro experiments confirmed the similarities between cTnI and LPS immunogenicity during stress. Also, although it could be argued that LPS would still elicit proinflammatory responses from $\mathrm{PM}_{\phi}$ under physiological state, a previous study and performed cytokine analysis (data not included) have demonstrated the exacerbation of these responses during CCS (Laukova et al., 2018).

In further in vitro investigations, we explored the inhibitory effects of ALX on GRK5 and/or the stimulatory effects of FSK on AC/cAMP synthesis for attenuating the LPSinduced inflammatory responses during stress. The followedup cytokine analysis demonstrated that only the inhibition of GRK5 during stress could not effectively attenuate the proinflammatory response elicited from the $\mathrm{PM}_{\phi}$, although the ALX dosage used was within the range reported previously to halt proinflammatory response in a different pathological model (Quan et al., 2019). Comparatively, FSK-AC/cAMP exerted more anti-inflammatory effects than ALX did; regardless, their combination showed the most potency at exhibiting adaptive immunoregulation. FSK enhanced cAMP bioavailability in single and combined treatments. Consistent with these findings, FSK-AC/cAMP had been reported earlier to exert anti-inflammatory effects via NF- $\kappa$ B inhibition (Chiadak et al., 2016). To validate the mechanism utilized by the ALX and FSK combination to attenuate the proinflammatory responses, immunofluorescence was used to ascertain the expressions of GRK5 among the in vitro treatment groups (Figures $\mathbf{2 G}, \mathbf{H}$ ). As suggested (Homan et al., 2014), ALX in both single and combination treatment inhibited GRK5 expression and nuclear translocation, while FSK failed to do either, just like the group without treatment interventions (LPS + ISO). However, although GRK5 translocated into the nuclei of LPS + ISO as much as it did in LPS + ISO + FSK, proinflammatory responses were not aggravated in the latter. As such, these were concluded from Figure 2 and the findings of others: (1) Although ALX inhibited GRK5-mediated proinflammatory response activation, other inflammatory mediators such as kinases, peptides, and amines are still capable of inducing the immune responses (Abdulkhaleq et al., 2018), as observed in the LPS + ISO + ALX group. (2) Also, the abolishment of cAMP-dependent adaptive immunoregulation by stress in the LPS + ISO + ALX group may have enabled the observed proinflammatory responses, as demonstrated previously (Bopp et al., 2009; Wehbi and Taskén, 2016). (3) Besides, unlike ALX, which attenuates GRK5-mediated inflammatory responses, FSK-ACs/cAMP adaptively regulates multiple anti-inflammatory cascades (Raker et al., 2016; Wehbi and Taskén, 2016). Hence, in line with previous studies (Bopp et al., 2009; Patial et al., 2011; Chiadak et al., 2016; Raker et al., 2016; Wehbi and Taskén, 2016), we confirmed that ALX and FSK combination attained their potency primarily via FSK/ACs/cAMP-mediated adaptive immunoregulation, coupled with ALX inhibiting the activation of maladaptive inflammatory responses via GRK5. Based on these findings, it was hypothesized that rather than either FSK or ALX treatment, their combination might attenuate myocardial inflammation, which drives the adverse remodeling of hearts during CCS.

These hypotheses were tested by translating ALX and/or FSK treatments in vivo during CCS. At the end of all models, echocardiogram results revealed that the ALX and FSK combination prevent LVSD during CCS, by maintaining typical heart rates, ejection fractions above $65 \%$, and fraction shortenings above $35 \%$. However, ALX treatment failed to maintain proper cardiac function during CCS, while FSK treatment resulted in arrhythmias. Contrary to our findings, Mo et al. (2020) reported that ALX improved cardiac function, but this was in acute myocardial infarction animal models and not during CCS or in an isoproterenol-induced cardiomyopathy model. Also, the ALX dosage they employed was 10 times more than what we used in this study. Nonetheless, our findings regarding FSK-induced arrhythmias were in conformity with the reports from previous 


\section{A}

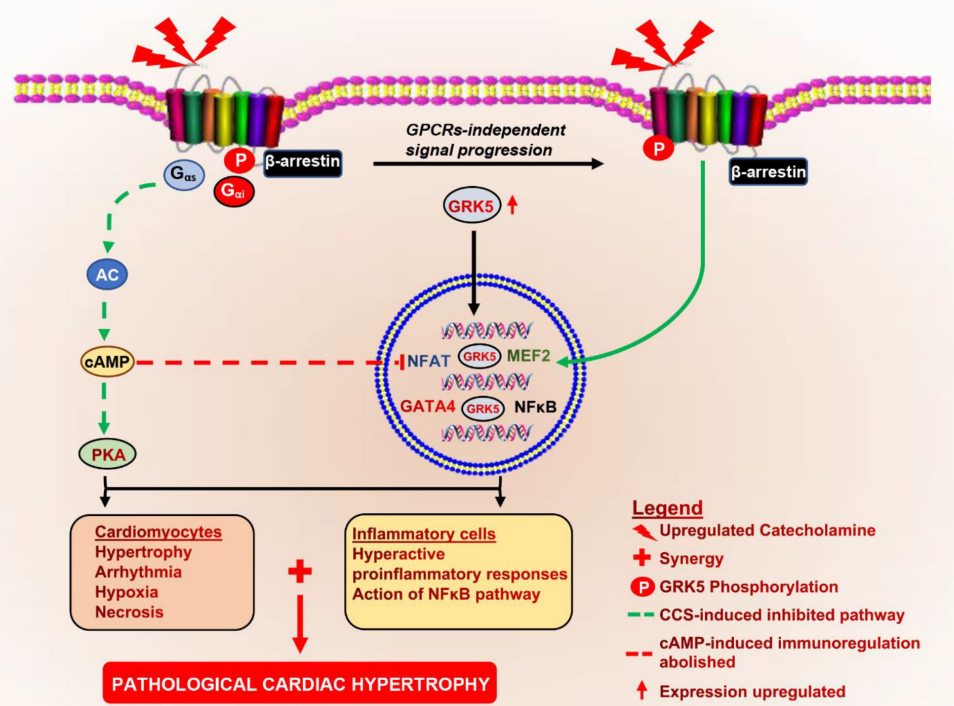

B
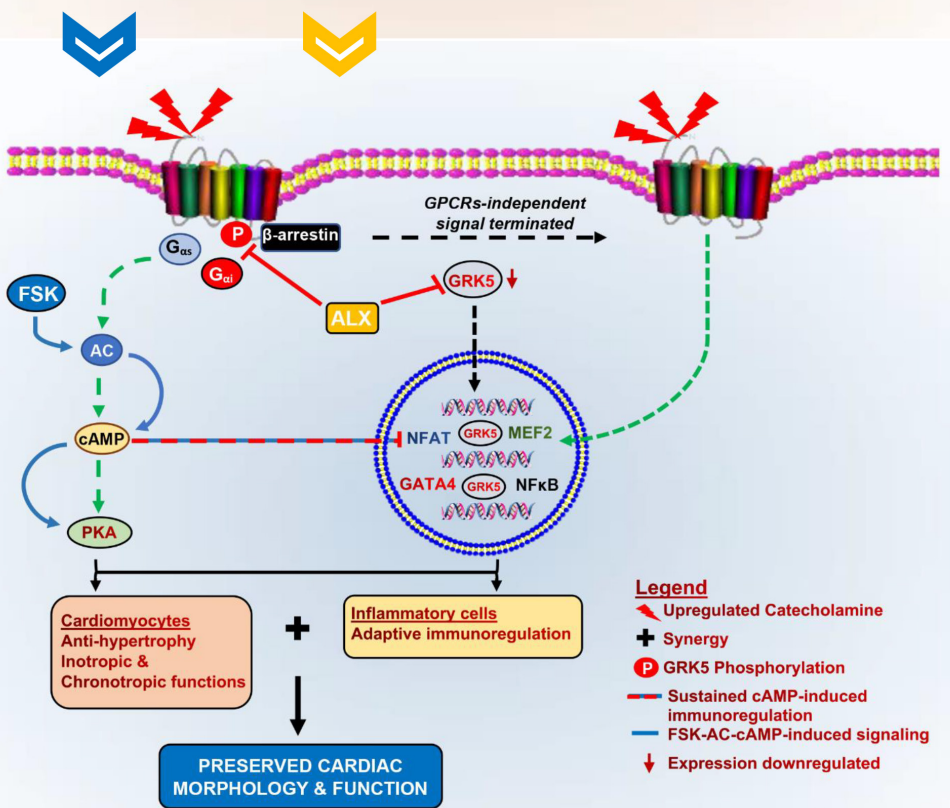

FIGURE 6 | Schematics of the pathomechanism of isoproterenol-induced cardiomyopathy and the mechanisms of ALX and FSK combination treatment. (A) During $\mathrm{CCS}, \mathrm{G}_{\alpha \mathrm{s}}-\mathrm{AC}$ is decoupled from $\beta A R s$, thereby inhibiting cAMP synthesis, which affects cardiac function and immunoregulation in the myocardia adversely. Also, GRK5 upregulates and phosphorylates $\beta A R s$ as well as translocations into the nuclei to induce GPCRs-independent stimuli signaling. The nuclear activity of GRK5 induces the activation of cardiomyocyte hypertrophy transcriptional factors. These result in excessive cardiomyocyte hypertrophy and hyperactive proinflammatory responses. At the same time, cAMP-mediated adaptive immunoregulation is abolished. The synergy of these cascades results in the exacerbation of collagen deposits which pathologically remodels the heart. (B) The administration of ALX and FSK combination facilitates ALX-GRK5 inhibition (expressions and nuclear translocation), which prevents cardiomyocyte hypertrophy; while, FSK-ACs-cAMP modulates adaptive immunoregulation and cardiac inotropic functions. Combining these mechanisms preserves cardiac morphology and prevents left ventricular systolic dysfunction (LVSD) during CCS.

studies (Christ et al., 2014). Intriguingly, tachyarrhythmia did not occur in the combined treatment group despite the presence of FSK, and we speculated that this might be due to an effect exerted by ALX.

Initially, in this study, the occurrence of LVSD was associated with alterations in cardiac and inflammatory proteins in the myocardia during CCS. As such, to confirm that the treatment interventions influenced their expression to preserve cardiac function, these proteins were reevaluated. In summary, the immunoblotting results showed that the ALX and FSK combination had normalized the expression of all the assessed cardiac and inflammatory proteins besides AC6, which is 
specifically responsible for cardiomyocyte protection during stress (Wu et al., 2017).

Although ALX treatment inhibited GRK5, the TFs, GATA4, NFAT, MEF2, and NF-кB were still overexpressed during CCS (Supplementary Figure 4). Consistent with these findings, Hullmann et al. (2014) had earlier suggested that the inhibition of GRK5 might not halt the activation of NFAT, which might have applied to GATA4, MEF2, and NF-KB as well. Contrarily, FSK single treatment decreased the expression of these TFs, just as Zoccarato et al. (2015) and Chiadak et al. (2016) had demonstrated.

Also, the myocardial morphometric data indicated that the hearts of PCH mice were hypertrophied and had marked fibrosis, which were determined with measured cardiomyocyte diameters, upregulated ANP and BNP upregulation, and Masson's-stained tissues. Further investigations with cleaved caspase- 3 and collagen I and III immunoblotting confirmed a significant increase in cardiomyocyte apoptosis and collagen depositions in PCH myocardial. Intriguingly, ALX and FSK combination attenuated excessive cardiomyocyte hypertrophy, apoptosis, and myocardial fibrosis during CCS. Meanwhile, neither ALX nor FSK treatments were able to achieve all the aforementioned individually. ALX treatment downregulated ANP and BNP and attenuated GRK5-induced hypertrophy as suggested (Homan et al., 2014), but it failed to prevent apoptosis, hence, the occurrence of fibrosis. Consistent with these findings, apoptosis has been well demonstrated to aggravate collagen deposition and fibrosis (Hinz and Lagares, 2020). In contrast to ALX treatment, FSK-AC-cAMP significantly inhibited cardiomyocyte apoptosis and fibrosis, just as demonstrated previously (Kwon et al., 2004; Lee et al., 2010; Younce et al., 2013). However, myocardial hypertrophy remained incompletely resolved by FSK treatment. Wolf et al. (2005) had previously reported that myocardial hypertrophy increases tachyarrhythmia susceptibility. Hence, this may have accounted for the observed tachyarrhythmia in the FSK treatment group but not in the ALX and FSK combination group since ALX had attenuated hypertrophy.

Lastly, assessing the effects of the treatment intervention on modulating myocardial inflammation during CCS showed that ALX and FSK combination prevents cardiomyocyte necrosis as sera concentrations of $\mathrm{cTnI}$ in mice from this group were significantly downregulated. Hence, cTnI-induced inflammation was attenuated in the ALX and FSK combination treatment group. Individually, in contrast with ALX, FSK-AC-cAMP significantly decreased cTnI (cardiomyocyte necrosis) during CSS, as demonstrated in other studies (Wang et al., 2016). Furthermore, in correlation with the extent of necrosis shown in PCH hearts, enormous amounts of $\mathrm{CD}^{+} 8^{+}$inflammatory cells were found to have infiltrated their myocardia. This provides supporting evidence that myocardial inflammation is exacerbated by stress, as demonstrated previously (Scally et al., 2019).

Nonetheless, we found that the ALX and FSK combination treatment was the most potent in minimizing $\mathrm{CD}^{+} 8^{+}$inflammatory cell infiltration into the myocardial during CCS, although FSK treatment also attained this significantly. Similarly, FSK-AC/cAMP has been shown to adaptively modulate myocardial inflammatory responses
(Lee et al., 2010; Shim et al., 2014). However, regarding ALX treatment, we found that it was not effective at decreasing myocardial inflammation, as Mo et al. (2020) had reported. The discrepancies in these findings may be due to the differences in the cardiovascular disease model being investigated and the variation in ALX dosage employed. To complement our findings, the evaluated cytokine concentrates depicted ALX and FSK combination treatment during CCS as the most effective intervention at keeping the gap between proinflammatory (IL-1 $\beta$, IL-6, and TNF $\alpha$ ) and anti-inflammatory (IL-10 and TGF- $\beta$ ) responses close to a homeostatic immune state. This certainly contributed to the prevention of biased prolonged proinflammatory responses, which could have exacerbated myocyte necrosis, apoptosis, and aggravated interstitial collagen deposits, as shown in other studies (Xiao et al., 2018; Sandstedt et al., 2019).

\section{CONCLUSION}

Here, we demonstrated the implication of maladaptive inflammatory response in the pathogenesis of isoproterenolinduced cardiomyopathy. Our findings showed that besides ALX preventing the occurrence of cardiomyocyte hypertrophy, it complemented the efforts of FSK-ACs/cAMP-mediated immunoregulation by abolishing GRK5-mediated induction of inflammatory responses. These combined efforts helped ALX and FSK maintain the myocardial homeostasis, thereby preserving the cardiac morphology and function during CCS (Figure 6). Owing to the clinical significance of this study, it is appropriate to acknowledge its limitations. It could be argued that ALX can inhibit IkB kinases and TANK-binding kinase 1 (TBK1) besides GRK5. Nonetheless, the inhibition of IkB kinases and TBK1 along with GRK5 by ALX, as demonstrated (Mo et al., 2020), still did not prevent the LVSD in PCH mice models. Also, further studies into determining the toxicity of ALX and FSK combined treatment and its impact on diastolic function and lung congestion during CCS will be required to fully ascertain their therapeutic and translational potentials.

\section{DATA AVAILABILITY STATEMENT}

The original contributions presented in the study are included in the article/Supplementary Material, further inquiries can be directed to the corresponding author/s.

\section{ETHICS STATEMENT}

The animal study was reviewed and approved by the Experimental Animal Centre of Xuzhou Medical University and the Animal Ethics Committee of the Medical University.

\section{AUTHOR CONTRIBUTIONS}

GKA conceived the experiments ideas. HS and GKA designed the experiments. GKA and $\mathrm{HH}$ isolated and cultured $\mathrm{PM}_{\phi}$. AA 
provided the experimental animals. RR assisted in making the animal models. SA and SK performed $\mathrm{PM}_{\phi}$ identification. KL, $\mathrm{Q}-\mathrm{MD}$, and XM performed the cardiac function assessments. GKA, TM, and JA-A performed the sera cytokine analysis. GKA, SA, Q-MD, RM, and WS analyzed and interpreted the data. GKA, RM, MN, JA-A, JM, and WS drafted and wrote the manuscript with contributions from all authors. All authors discussed, proofread, and approved the manuscript.

\section{FUNDING}

The following grants funded this study; National Natural Science Foundation of China (Nos. 81461138036 and 81370329), Postgraduate Research and Practice Innovation Program of Jiangsu Province, China (KYCX17-1712), and Priority

\section{REFERENCES}

Abdulkhaleq, L. A., Assi, M. A., Abdullah, R., Zamri-Saad, M., Taufiq-Yap, Y. H., and Hezmee, M. N. M. (2018). The crucial roles of inflammatory mediators in inflammation: a review. Vet. World 11, 627-635. doi: 10.14202/vetworld.2018. 627-635

Adzika, G. K., Machuki, J. O., Shang, W., Hou, H., Ma, T., Wu, L., et al. (2019). Pathological cardiac hypertrophy: the synergy of adenylyl cyclases inhibition in cardiac and immune cells during chronic catecholamine stress. J. Mol. Med. 97, 897-907. doi: 10.1007/s00109-019-01790-0

Bopp, T., Dehzad, N., Reuter, S., Klein, M., Ullrich, N., Stassen, M., et al. (2009). Inhibition of cAMP degradation improves regulatory T cell-mediated suppression. J. Immunol. 182, 4017-4024. doi: 10.4049/jimmunol.0803310

Cai, Z. L., Shen, B., Yuan, Y., Liu, C., Xie, Q. W., Hu, T. T., et al. (2020). The effect of HMGA1 in LPS-induced Myocardial Inflammation. Int. J. Biol. Sci. 16, 1798-1810. doi: 10.7150/ijbs.39947

Chiadak, J. D., Arsenijevic, T., Verstrepen, K., Gregoire, F., Bolaky, N., Delforge, V., et al. (2016). Forskolin Inhibits Lipopolysaccharide-Induced Modulation of MCP-1 and GPR120 in 3T3-L1 Adipocytes through an Inhibition of NFKB. Mediators Inflamm. 2016:1431789.

Christ, T., Rozmaritsa, N., Engel, A., Berk, E., Knaut, M., Metzner, K., et al. (2014). Arrhythmias, elicited by catecholamines and serotonin, vanish in human chronic atrial fibrillation. Proc. Natl. Acad. Sci. U.S.A. 111, 11193-11198. doi: $10.1073 /$ pnas.1324132111

Frey, N., Katus, H. A., Olson, E. N., and Hill, J. A. (2004). Hypertrophy of the heart: A new therapeutic target? Circulation 109, 1580-1589. doi: 10.1161/01. cir.0000120390.68287.bb

Galati, G., Leone, O., Pasquale, F., Olivotto, I., Biagini, E., Grigioni, F., et al. (2016). Histological and histometric characterization of myocardial fibrosis in end-stage hypertrophic cardiomyopathy: a clinical-pathological study of 30 Explanted Hearts. Circ. Heart Fail. 9:e003090. doi: 10.1161/circheartfailure.116. 003090

Gold, J. I., Gao, E., Shang, X., Premont, R. T., and Koch, W. J. (2012). Determining the absolute requirement of $\mathrm{G}$ protein-coupled receptor kinase 5 for pathological cardiac hypertrophy: short communication. Circ. Res. 111, 1048-1053. doi: 10.1161/circresaha.112.273367

Gouveia, K., and Hurst, J. L. (2019). Improving the practicality of using nonaversive handling methods to reduce background stress and anxiety in laboratory mice. Sci. Rep. 9:20305. doi: 10.1038/s41598-019-56860-7

Haider, K. H., and Stimson, W. H. (1993). Cardiac troponin-I: a biochemical marker for cardiac cell necrosis. Dis. Markers 11, 205-215. doi: 10.1155/1993/ 901687

Hartupee, J., and Mann, D. L. (2016). Role of inflammatory cells in fibroblast activation. J. Mol. Cell. Cardiol. 93, 143-148. doi: 10.1016/j.yjmcc.2015.11.016

Heidt, T., Courties, G., Dutta, P., Sager, H. B., Sebas, M., Iwamoto, Y., et al. (2014). Differential contribution of monocytes to heart macrophages in steady-state and after myocardial infarction. Circ. Res. 115, 284-295. doi: 10.1161/circresaha. 115.303567
Academic Program Development of Jiangsu Higher Education Institutions (PAPD).

\section{ACKNOWLEDGMENTS}

We appreciate the inputs of $\mathrm{Lu} \mathrm{Fu}$ during the assessment of cardiac functions.

\section{SUPPLEMENTARY MATERIAL}

The Supplementary Material for this article can be found online at: https://www.frontiersin.org/articles/10.3389/fcell.2021. 719351/full\#supplementary-material

Hinz, B., and Lagares, D. (2020). Evasion of apoptosis by myofibroblasts: a hallmark of fibrotic diseases. Nat. Rev. Rheumatol. 16, 11-31. doi: 10.1038/s41584-0190324-5

Homan, K. T., Wu, E., Cannavo, A., Koch, W. J., and Tesmer, J. J. (2014). Identification and characterization of amlexanox as a $\mathrm{G}$ protein-coupled receptor kinase 5 inhibitor. Molecules 19, 16937-16949. doi: 10.3390/ molecules191016937

Huang, X. D., and Wong, T. M. (1989). Arrhythmogenic effect of forskolin in the isolated perfused rat heart: influence of nifedipine or reduction of external calcium [corrected]. Clin. Exp. Pharmacol. Physiol. 16, 751-757. doi: 10.1111/j. 1440-1681.1989.tb01513.x

Hullmann, J. E., Grisanti, L. A., Makarewich, C. A., Gao, E., Gold, J. I., Chuprun, J. K., et al. (2014). GRK5-mediated exacerbation of pathological cardiac hypertrophy involves facilitation of nuclear NFAT activity. Circ. Res. 115, 976-985. doi: 10.1161/circresaha.116.304475

Hulsmans, M., Sam, F., and Nahrendorf, M. (2016). Monocyte and macrophage contributions to cardiac remodeling. J. Mol. Cell. Cardiol. 93, 149-155. doi: 10.1016/j.yjmcc.2015.11.015

Islam, K. N., Bae, J. W., Gao, E., and Koch, W. J. (2013). Regulation of nuclear factor $\kappa \mathrm{B}(\mathrm{NF}-\kappa \mathrm{B})$ in the nucleus of cardiomyocytes by $\mathrm{G}$ protein-coupled receptor kinase 5 (GRK5). J. Biol. Chem. 288, 35683-35689. doi: 10.1074/jbc. M113.529347

Jia, D., Jiang, H., Weng, X., Wu, J., Bai, P., Yang, W., et al. (2019). Interleukin-35 promotes macrophage survival and improves wound healing after myocardial infarction in mice. Circ. Res. 124, 1323-1336. doi: 10.1161/circresaha.118. 314569

Kaya, Z., Göser, S., Buss, S. J., Leuschner, F., Ottl, R., Li, J., et al. (2008). Identification of cardiac troponin I sequence motifs leading to heart failure by induction of myocardial inflammation and fibrosis. Circulation 118, 2063-2072. doi: 10.1161/circulationaha.108.788711

Kaya, Z., Katus, H. A., and Rose, N. R. (2010). Cardiac troponins and autoimmunity: their role in the pathogenesis of myocarditis and of heart failure. Clin. Immunol. 134, 80-88. doi: 10.1016/j.clim.2009.04.008

Kwon, G., Pappan, K. L., Marshall, C. A., Schaffer, J. E., and McDaniel, M. L. (2004). cAMP Dose-dependently prevents palmitate-induced apoptosis by both protein kinase A- and cAMP-guanine nucleotide exchange factor-dependent pathways in beta-cells. J. Biol. Chem. 279, 8938-8945. doi: 10.1074/jbc.M31033 0200

Laukova, M., Vargovic, P., Rokytova, I., Manz, G., and Kvetnansky, R. (2018). Repeated stress exaggerates lipopolysaccharide-induced inflammatory response in the rat spleen. Cell. Mol. Neurobiol. 38, 195-208. doi: 10.1007/s10571-0170546-5

Lee, J. Y., Kim, J. H., Chae, G., Lee, B. K., Ha, K. S., Kwon, Y. G., et al. (2010). Cyclic AMP prolongs graft survival by suppressing apoptosis and inflammatory gene expression in acute cardiac allograft rejection. Exp. Mol. Med. 42, 69-79. doi: 10.3858/emm.2010.42.1.008

Lin, Y., Zhang, X., Xiao, W., Li, B., Wang, J., Jin, L., et al. (2016). Endoplasmic reticulum stress is involved in DFMO attenuating isoproterenol-induced 
cardiac hypertrophy in rats. Cell. Physiol. Biochem. 38, 1553-1562. doi: 10.1159/ 000443096

Liu, W., Wang, X., Mei, Z., Gong, J., Gao, X., Zhao, Y., et al. (2015). Chronic stress promotes the progression of pressure overload-induced cardiac dysfunction through inducing more apoptosis and fibrosis. Physiol. Res. 64, 325-334. doi: 10.33549/physiolres. 932778

Marstrand, P., Han, L., Day Sharlene, M., Olivotto, I., Ashley Euan, A., Michels, M., et al. (2020). Hypertrophic cardiomyopathy with left ventricular systolic dysfunction. Circulation 141, 1371-1383. doi: 10.1161/CIRCULATIONAHA. 119.044366

Mo, C., Ha, H., Tang, X., Lu, X., Wei, Y., Luo, D., et al. (2020). Protein kinase TBK1/IKK $\varepsilon$ inhibitor Amlexanox improves cardiac function after acute myocardial infarction in rats. Panminerva Med. doi: 10.23736/s0031-0808.20. 03937-3 [Epub ahead of print].

Murphy, J. G., Crosby, K. C., Dittmer, P. J., Sather, W. A., and Dell'Acqua, M. L. (2019). AKAP79/150 recruits the transcription factor NFAT to regulate signaling to the nucleus by neuronal L-type $\mathrm{Ca}(2+)$ channels. Mol. Biol. Cell 30, 1743-1756. doi: 10.1091/mbc.E19-01-0060

Patial, S., Shahi, S., Saini, Y., Lee, T., Packiriswamy, N., Appledorn, D. M., et al. (2011). G-protein coupled receptor kinase 5 mediates lipopolysaccharideinduced NFKB activation in primary macrophages and modulates inflammation in vivo in mice. J. Cell. Physiol. 226, 1323-1333. doi: 10.1002/jcp.22460

Paur, H., Wright, P. T., Sikkel, M. B., Tranter, M. H., Mansfield, C., O'Gara, P., et al. (2012). High levels of circulating epinephrine trigger apical cardiodepression in a $\beta 2$-adrenergic receptor/Gi-dependent manner: a new model of Takotsubo cardiomyopathy. Circulation 126, 697-706. doi: 10.1161/circulationaha.112. 111591

Pereira, L., Rehmann, H., Lao, D. H., Erickson, J. R., Bossuyt, J., Chen, J., et al. (2015). Novel Epac fluorescent ligand reveals distinct Epac1 vs. Epac2 distribution and function in cardiomyocytes. Proc. Natl. Acad. Sci. U.S.A. 112, 3991-3996. doi: 10.1073/pnas.1416163112

Plattner, F., Hayashi, K., Hernández, A., Benavides, D. R., Tassin, T. C., Tan, C., et al. (2015). The role of ventral striatal cAMP signaling in stress-induced behaviors. Nat. Neurosci. 18, 1094-1100. doi: 10.1038/nn.4066

Qi, L., Chi, X., Zhang, X., Feng, X., Chu, W., Zhang, S., et al. (2019). Kindlin-2 suppresses transcription factor GATA4 through interaction with SUV39H1 to attenuate hypertrophy. Cell Death Dis. 10:890. doi: 10.1038/s41419-019-21210

Quan, M. Y., Song, X. J., Liu, H. J., Deng, X. H., Hou, H. Q., Chen, L. P., et al. (2019). Amlexanox attenuates experimental autoimmune encephalomyelitis by inhibiting dendritic cell maturation and reprogramming effector and regulatory T cell responses. J. Neuroinflammation 16:52. doi: 10.1186/s12974-019-1438-Z

Raker, V. K., Becker, C., and Steinbrink, K. (2016). The cAMP pathway as therapeutic target in autoimmune and inflammatory diseases. Front. Immunol. 7:123. doi: $10.3389 /$ fimmu. 2016.00123

Ray, A., and Dittel, B. N. (2010). Isolation of mouse peritoneal cavity cells. J. Vis. Exp. 35:1488. doi: 10.3791/1488

Sandstedt, J., Sandstedt, M., Lundqvist, A., Jansson, M., Sopasakis, V. R., Jeppsson, A., et al. (2019). Human cardiac fibroblasts isolated from patients with severe heart failure are immune-competent cells mediating an inflammatory response. Cytokine 113, 319-325. doi: 10.1016/j.cyto.2018.09.021

Scally, C., Abbas, H., Ahearn, T., Srinivasan, J., Mezincescu, A., Rudd, A., et al. (2019). Myocardial and systemic inflammation in acute stress-induced (Takotsubo) Cardiomyopathy. Circulation 139, 1581-1592. doi: 10.1161/ circulationaha.118.037975

Schaeuble, D., Packard, A. E. B., McKlveen, J. M., Morano, R., Fourman, S., Smith, B. L., et al. (2019). Prefrontal cortex regulates chronic stress-induced cardiovascular susceptibility. J. Am. Heart Assoc. 8:e14451. doi: 10.1161/jaha. 119.014451

Shim, S. H., Kim, D. S., Cho, W., and Nam, J. H. (2014). Coxsackievirus B3 regulates T-cell infiltration into the heart by lymphocyte function-associated antigen-1 activation via the cAMP/Rap1 axis. J. Gen. Virol. 95(Pt 9), 2010-2018. doi: 10.1099/vir.0.065755-0

Spadari, R. C., Cavadas, C., de Carvalho, A., Ortolani, D., de Moura, A. L., and Vassalo, P. F. (2018). Role of Beta-adrenergic receptors and Sirtuin signaling in the heart during aging, heart failure, and adaptation to stress. Cell. Mol. Neurobiol. 38, 109-120. doi: 10.1007/s10571-017-0557-2

Sun, X., Zhou, M., Wen, G., Huang, Y., Wu, J., Peng, L., et al. (2021). Paroxetine attenuates cardiac hypertrophy via blocking GRK2 and ADRB1 Interaction in Hypertension. J. Am. Heart Assoc. 10:e016364. doi: 10.1161/jaha.120.016364
Traynham, C. J., Hullmann, J., and Koch, W. J. (2016). Canonical and noncanonical actions of GRK5 in the heart. J. Mol. Cell. Cardiol. 92, 196-202. doi: 10.1016/j.yjmcc.2016.01.027

Wang, Z., Liu, D., Varin, A., Nicolas, V., Courilleau, D., Mateo, P., et al. (2016). A cardiac mitochondrial cAMP signaling pathway regulates calcium accumulation, permeability transition and cell death. Cell Death Dis. 7:e2198. doi: $10.1038 /$ cddis.2016.106

Wehbi, V. L., and Taskén, K. (2016). Molecular mechanisms for cAMP-mediated immunoregulation in T cells - role of anchored protein kinase A signaling units. Front. Immunol. 7:222. doi: 10.3389/fimmu.2016.00222

Weisheit, C. K., Kleiner, J. L., Rodrigo, M. B., Niepmann, S. T., Zimmer, S., Duerr, G. D., et al. (2021). CX3CR1 is a prerequisite for the development of cardiac hypertrophy and left ventricular dysfunction in mice upon transverse aortic constriction. PLoS One 16:e243788. doi: 10.1371/journal.pone.0243788

Whelan, R. S., Kaplinskiy, V., and Kitsis, R. N. (2010). Cell death in the pathogenesis of heart disease: mechanisms and significance. Annu. Rev. Physiol. 72, 19-44. doi: 10.1146/annurev.physiol.010908.163111

Wolf, C. M., Moskowitz, I. P., Arno, S., Branco, D. M., Semsarian, C., Bernstein, S. A., et al. (2005). Somatic events modify hypertrophic cardiomyopathy pathology and link hypertrophy to arrhythmia. Proc. Natl. Acad. Sci. U.S.A. 102, 18123-18128. doi: 10.1073/pnas.0509145102

Wu, Y. S., Chen, C. C., Chien, C. L., Lai, H. L., Jiang, S. T., Chen, Y. C., et al. (2017). The type VI adenylyl cyclase protects cardiomyocytes from $\beta$-adrenergic stress by a PKA/STAT3-dependent pathway. J. Biomed. Sci. 24:68. doi: 10.1186/ s12929-017-0367-3

Xiao, H., Li, H., Wang, J. J., Zhang, J. S., Shen, J., An, X. B., et al. (2018). IL-18 cleavage triggers cardiac inflammation and fibrosis upon $\beta$-adrenergic insult. Eur. Heart J. 39, 60-69. doi: 10.1093/eurheartj/ehx261

Xie, M., Burchfield, J. S., and Hill, J. A. (2013). Pathological ventricular remodeling: therapies: part 2 of 2. Circulation 128, 1021-1030. doi: 10.1161/circulationaha. 113.001879

Younce, C. W., Burmeister, M. A., and Ayala, J. E. (2013). Exendin-4 attenuates high glucose-induced cardiomyocyte apoptosis via inhibition of endoplasmic reticulum stress and activation of SERCA2a. Am. J. Physiol. Cell Physiol. 304, C508-C518. doi: 10.1152/ajpcell.00248.2012

Zhang, P., Li, T., Liu, Y. Q., Zhang, H., Xue, S. M., Li, G., et al. (2019). Contribution of DNA methylation in chronic stress-induced cardiac remodeling and arrhythmias in mice. FASEB J. 33, 12240-12252. doi: 10.1096/fj.201900100R

Zhao, C. H., Ma, X., Guo, H. Y., Li, P., and Liu, H. Y. (2017). RIP2 deficiency attenuates cardiac hypertrophy, inflammation and fibrosis in pressure overload induced mice. Biochem. Biophys. Res. Commun. 493, 1151-1158. doi: 10.1016/j. bbrc.2017.07.035

Zhou, R., Ma, P., Xiong, A., Xu, Y., Wang, Y., and Xu, Q. (2017). Protective effects of low-dose rosuvastatin on isoproterenol-induced chronic heart failure in rats by regulation of DDAH-ADMA-NO pathway. Cardiovasc. Ther. 35:e12241. doi: 10.1111/1755-5922.12241

Zoccarato, A., Surdo, N. C., Aronsen, J. M., Fields, L. A., Mancuso, L., Dodoni, G., et al. (2015). Cardiac Hypertrophy Is Inhibited by a Local Pool of cAMP Regulated by Phosphodiesterase 2. Circ. Res. 117, 707-719. doi: 10.1161/ circresaha.114.305892

Conflict of Interest: The authors declare that the research was conducted in the absence of any commercial or financial relationships that could be construed as a potential conflict of interest.

Publisher's Note: All claims expressed in this article are solely those of the authors and do not necessarily represent those of their affiliated organizations, or those of the publisher, the editors and the reviewers. Any product that may be evaluated in this article, or claim that may be made by its manufacturer, is not guaranteed or endorsed by the publisher.

Copyright $\odot 2021$ Adzika, Hou, Adekunle, Rizvi, Adzraku, Li, Deng, Mprah, Ndzie Noah, Adu-Amankwaah, Machuki, Shang, Ma, Koda, Ma and Sun. This is an openaccess article distributed under the terms of the Creative Commons Attribution License (CC BY). The use, distribution or reproduction in other forums is permitted, provided the original author(s) and the copyright owner(s) are credited and that the original publication in this journal is cited, in accordance with accepted academic practice. No use, distribution or reproduction is permitted which does not comply with these terms. 